\title{
In vitro and in vivo efficacy study of cefepime, doripenem, tigecycline, and tetracycline against extended-spectrum beta-lactamases Escherichia coli in chickens
}

\author{
Yaser Hamadeh Tarazi $^{1}$, Ehab A. Abu-Basha ${ }^{1}$, Zuhair Bani Ismail ${ }^{2}$ and Rawan A. Tailony ${ }^{1}$
}

1. Department of Basic Medical Veterinary Sciences, Faculty of Veterinary Medicine, Jordan University of Science and Technology, Irbid, Jordan; 2. Department of Veterinary Clinical Sciences, Faculty of Veterinary Medicine, Jordan University of Science and Technology, Irbid, Jordan.

Corresponding author: Yaser Hamadeh Tarazi, e-mail: tarazi@just.edu.jo

Co-authors: EAA: abubasha@just.edu.jo, ZBI: zuhair72@just.edu.jo, RAT: tailony.rawan@yahoo.com

Received: 08-09-2019, Accepted: 21-01-2020, Published online: 11-03-2020

doi: www.doi.org/10.14202/vetworld.2020.446-451 How to cite this article: Tarazi YH, Abu-Basha EA, Ismail ZB, Tailony RA (2020) In vitro and in vivo efficacy study of cefepime, doripenem, tigecycline, and tetracycline against extended-spectrum beta-lactamases Escherichia coli in chickens, Veterinary World, 13(3): 446-451.

\begin{abstract}
Background and Aim: At present, there are no data about the efficacy of some recent antibiotics on Escherichia coli in broiler chickens in the study area. This study was designed to evaluate the in vitro and in vivo efficacy of cefepime, doripenem, tigecycline, and tetracycline against multidrug-resistant-extended-spectrum beta-lactamases (MDR-ESBLs) producing $E$. coli in broiler chicks.

Materials and Methods: A total of 34 MDR-ESBLs E. coli isolates were used in this study. In vitro evaluation of the antibacterial efficacy of cefepime, doripenem, tigecycline, and tetracycline were performed using disk diffusion and minimum inhibitory concentration (MIC) assays. In vivo evaluation of the efficacy of the antibiotics was perfumed using 180, 2-week-old chicks challenged with MDR-ESBL-producing E. coli strain O78. Chicks were divided into six groups (30 chicks each) according to the treatment regimen. Treatment was administered to chicks in Groups 3-6 intravenously, twice per day for 1 week using one antibiotic per group at concentration 10 times the determined MIC. Chicks in the positive control (Group 1) were challenged and received $0.2 \mathrm{ml}$ of sterile Tryptone Soy Broth (TSB), while those in the negative control (Group 2) were not challenged and received $0.2 \mathrm{ml}$ of sterile TSB. The severity of clinical signs, gross lesions, and mortality rate was scored and compared between groups.

Results: All E. coli isolates were sensitive to doripenem and tigecycline, while $88 \%$ were sensitive to cefepime and only $23 \%$ were sensitive to tetracycline. In vivo antibiotic efficacy evaluation in challenged chicks revealed a significant reduction in the severity of clinical signs, gross lesions, and mortality $(3 \%)$ in chicks treated with cefepime compared to non-treated chicks (55\%). There was no significant effect on the severity of clinical signs, gross lesions, and mortality in chicks treated with doripenem, tigecycline, and tetracycline compared to non-treated chicks. The mortality rates of chicks treated with doripenem, tigecycline, and tetracycline were $57 \%, 50 \%$, and $90 \%$, respectively.

Conclusion: The results of this study indicate that most MDR-ESBLs producing $E$. coli isolates were sensitive to doripenem, tigecycline, and cefepime. However, in vivo study indicated that only cefepime was effective and resulted in a significant reduction in clinical signs, gross lesions, and mortality in infected chicks. Therefore, cefepime could be used to treat naturally infected chickens with MDR-ESBLs producing strains of E. coli.
\end{abstract}

Keywords: antimicrobial resistance, chickens, colibacillosis, Escherichia coli, multidrug-resistant-extended-spectrum beta-lactamases.

\section{Introduction}

The emergence of infectious diseases caused by multidrug-resistant (MDR) pathogens has become a serious global health problem with significant increase in morbidity and mortality $[1,2]$. In recent years, many life-threatening diseases caused by methicillin-resistant Staphylococcus aureus, extended-spectrum beta-lactamases (ESBLs) producing Escherichia coli,

Copyright: Tarazi, et al. Open Access. This article is distributed under the terms of the Creative Commons Attribution 4.0 International License (http://creativecommons.org/licenses/by/4.0/), which permits unrestricted use, distribution, and reproduction in any medium, provided you give appropriate credit to the original author(s) and the source, provide a link to the Creative Commons license, and indicate if changes were made. The Creative Commons Public Domain Dedication waiver (http://creativecommons.org/ publicdomain/zero/1.0/) applies to the data made available in this article, unless otherwise stated. and MDR strains of Salmonella spp., Klebsiella spp., and Pseudomonas aeruginosa have become prevalent causing significant suffering in both humans and animals [3,4].

MDR E. coli is of particular concern because it is the most common Gram-negative opportunistic pathogen in humans and animals, causing urinary tract infections, diarrhea, and community- and hospital-acquired bacteremia [5]. ESBLs production by E. coli is responsible for resistance of the bacteria against a multitude of antimicrobial agents, mainly members of the cephalosporin and penicillin families [6]. Beta-lactamase hydrolyzes the $\beta$-lactam ring in these antibiotics rendering them ineffective [7]. ESBLs producing $E$. coli is considered a public health threat due to the risk of plasmid transfer of the ESBLs resistance genes to human pathogens from E. coli strains of animal origins $[6,7]$. 
The risks of antibiotic resistance spread among human and animal pathogens are seriously concerning to global health officials [8]. Although this process cannot be completely prevented, it can be slowed down through implementing strict antibiotic use regulations that ensure the judicious use of available antibiotics both in humans and animals and the development of new and effective antimicrobial agents [9-17].

We are running out of options to treat bacterial diseases due to widespread of antimicrobial resistance in poultry, the use of recent antibiotics could be a good option for treating bacterial diseases caused by MDRESBLs producing E. coli in chickens [18-21].

This study was designed to investigate the in vitro and in vivo efficacy of cefepime, doripenem, tigecycline, and tetracycline against MDR-ESBLs E. coli isolates in chicks.

\section{Materials and Methods}

\section{Ethical approval}

This study was reviewed and approved by the Institutional Animal Care and Use in research at Jordan University of Science and Technology (ACUC, Project \# 2019/48).

\section{Bacterial culture and preparation}

A total of 34 MDR-ESBLs producing E. coli isolates were used in this study. The bacteria were isolated from clinical samples obtained from naturally infected chickens and accumulated in Microbiology Research Laboratory from the previous study. E. coli isolates and serotype $\mathrm{O} 78$ used in the challenge assay in this study showed MDR pattern against $>11$ antimicrobial agents and was accumulated in Microbiology Research Laboratory, Faculty of Veterinary Medicine, Irbid-Jordan from the previous study. The isolates considered as ESBLs producer because they are resistant to penicillin, cephalosporin, and aztreonam and confirmed by double-disk synergy test as demonstrated in the materials and methods in this study.

The isolates were cultured on MacConkey and eosin methylene blue agar plates, incubated at $37^{\circ} \mathrm{C}$ for $24 \mathrm{~h}$. Bacterial identification was carried out based on colony morphology, Gram's stain characteristics, and biochemical characteristics including oxidase test and indole, methyl red, Voges-Proskauer, and citrate test[22].

\section{Antimicrobial agent's preparation}

Raw materials of antibiotics of doripenem, tigecycline, and tetracycline were provided by Cayman Chemical Company (Michigan, USA) and cefepime was provided by Demo S.A. Pharmaceutical (Votanikos, Greece). Doripenem and cefepime were suspended in saline while tigecycline and tetracycline were suspended in distilled water. The antibiotic disks of tigecycline $(15 \mu \mathrm{g})$, doripenem $(10 \mu \mathrm{g})$, oxacillin $(1 \mu \mathrm{g})$, and cefoxitin $(30 \mu \mathrm{g})$ were provided by Oxoid (Hampshire, UK), while cefepime $(30 \mu \mathrm{g})$ and tetracycline $(30 \mu \mathrm{g})$ were provided by
Bioanalyse (Ankara, Turkey). The antibiotic E-strips of tigecycline $(0.016-256 \mu \mathrm{g} / \mathrm{ml})$, tetracycline $(0.016-256 \mu \mathrm{g} / \mathrm{ml})$, cefepime $(0.016-256 \mu \mathrm{g} / \mathrm{ml})$, oxacillin $(0.016-256 \mu \mathrm{g} / \mathrm{ml})$, cefoxitin $(0.016-256 \mu \mathrm{g} / \mathrm{ml})$, and doripenem $(0.002-32 \mu \mathrm{g} / \mathrm{ml})$ were provided by HiMedia (Mumbai, India).

\section{Antimicrobial sensitivity test}

The antimicrobial sensitivity test was performed using the Kirby-Bauer disk diffusion method on Mueller-Hinton agar (Oxoid, UK). The fresh culture of ESBLs E. coli was diluted in normal saline to 0.5 McFarland standards $\left(\sim 1 \times 10^{8} \mathrm{CFU} / \mathrm{ml}\right)$. From this suspension, the surface of Mueller-Hinton agar plates was swabbed in four directions and the plates were left to dry and then the antibiotic discs were added. The plates were then incubated at $37^{\circ} \mathrm{C}$ for $24 \mathrm{~h}$ and the inhibition zones were recorded[23].

\section{Minimum inhibitory concentration (MIC)}

The MIC was performed using E-strip (Epsilometer; BioMérieux, France) according to the manufacturer's recommendations. The inoculum was prepared in Mueller-Hinton agar broth (Oxoid, UK) diluted to a $0.5 \mathrm{McF}$ arland standard. Then, the antibiotic strip was placed on the surface of Mueller-Hinton agar plate and incubated at $37^{\circ} \mathrm{C}$ for $24 \mathrm{~h}$. The MIC values were recorded at the points of intersection of the inhibition zones with the graded strips [23].

\section{ESBLs production confirmation by $E$. coli}

The production of ESBLs by the isolated $E$. coli strains used in this study was confirmed by double-disk synergy test[24]. All resistant strains against third-generation cephalosporin were used to detect ESBL production. Briefly, a disk containing cefotaxime $(30 \mu \mathrm{g})$ was placed at $15 \mathrm{~mm}$ away from a centrally placed disk containing amoxicillin-clavulanic acid $(20 \mu \mathrm{g} / 10 \mu \mathrm{g})$. The plates were then incubated at $37^{\circ} \mathrm{C}$ for $24 \mathrm{~h}$. The isolate was considered ESBLs producer if it showed a distinctive inhibition zone potentiated by amoxicillin/clavulanic acid disk $[24,25]$.

\section{E. coli pathogenicity assay}

The pathogenicity test aimed to determine which of the isolates is capable of causing the highest mortality to be used in the subsequent chick challenge test. Three O78 E. coli isolates were among the 34 MDRESBLs producing E. coli were used to evaluate pathogenicity by challenging 10, 2-week-old chicks by intrathoracic inoculation of the bacteria. Each chick received approximately $0.2 \mathrm{ml}$ inoculum $\left(1 \times 10^{9} \mathrm{CFU}\right.$ E. coli $/ \mathrm{ml}$ ) was injected into the left caudal thoracic air sac $[26,27]$. The chicks were monitored daily for 7 days and the mortality rate was calculated.

\section{E. coli challenge assay}

One hundred and eighty, 2-week-old chicks (Hubbard Classical) were obtained from a local company (Amman, Jordan) and maintained in the animal house, Faculty of Veterinary Medicine, Jordan University of Science and Technology. Chicks were 
housed in cages at room temperature of $27-29^{\circ} \mathrm{C}$. Freshwater and a commercial broiler chick feed were provided ad libitum. The feed was free of antimicrobial and anticoccidial drugs. Birds were provided with a continuous lighting pattern for $24 \mathrm{~h}$ during the experiment.

The chicks were divided into six groups $(30$ chicks each). In Group 1, chicks were infected and not treated (positive control); while in Group 2, the chicks were not infected and not treated control. In Groups 3-6, chicks were infected and treated with doripenem, cefepime, tigecycline, and tetracycline, respectively[28]. The antibiotics were administered at a dose rate equivalent to 10 times the $\mathrm{MIC}$, intravenously in the wing vein twice daily for a week. Chicks in the control groups received $0.2 \mathrm{ml}$ of sterile Tryptone Soy Broth intravenously in a manner similar to the chicks receiving antibiotics treatment.

Before inoculation, chicks were allowed to acclimatize in their environment for 1 week. The chicks were monitored daily during this period to determine their health status. After the challenge, chicks were monitored daily for expected clinical signs including depression, reluctance to move, gasping, and difficult breathing. The number of dead chicks was recorded daily for 7 days. On day 8 after inoculation, all chicks were humanely euthanized by cervical dislocation and a thorough necropsy was performed[29]. Gross lesions involving the major abdominal and thoracic organs, including the liver, heart, and air sacs, were reported and scored according to previously published methods [30].

\section{Statistical analysis}

The mean ranks of gross lesions involving different body organs were determined using KruskalWallis test. Statistical differences in the scores of gross lesions in different body organs between different treatment groups were analyzed using MannWhitney U-test. Statistical analysis was performed using the SPSS software version 24 (IBM, NY, USA). Statistical difference was considered statistically significant at $\mathrm{p} \leq 0.05$.

\section{Results}

\section{Antimicrobial sensitivity test}

The results of the disk diffusion sensitivity test and MIC of cefepime, doripenem, tigecycline, and tetracycline against MDR-ESBLs producing $E$. coli are presented in Figure-1. According to the disk diffusion test, all E. coli isolates were sensitive to doripenem and tigecycline while $88 \%$ were sensitive to cefepime and only $23 \%$ were sensitive to tetracycline. The MIC results showed that most $E$. coli isolates were more sensitive to doripenem and cefepime antibiotics.

The three MDR-ESBLs producing E. coli isolate used in pathogenicity assay were of serotype $\mathrm{O} 78$ isolated from naturally infected broiler chickens from the previous study. The isolates were sensitive to cefepime, doripenem, and tigecycline and resistant to tetracycline.

\section{Clinical signs and postmortem lesions}

Chicks in the negative control group showed no apparent clinical signs or postmortem lesions (Figure-2). In the positive control group, the chicks showed severe depression, fatigue, reluctance to move, and respiratory signs with a mortality rate of $55 \%$. Gross lesions were consistent with the formation of fibrinous material on the heart, liver, and air sacs (Figure-3). In Group 4 (infected and treated with cefepime), chicks showed no apparent clinical signs and only one chick died out of 30 (3\% mortality rate). Gross lesions were slight liver congestion and little fibrinous material accumulation on the heart with cloudy air sac (Figure-4). In Group 3 (infected and treated with doripenem), the clinical signs were similar to that of the positive control group. Seventeen chicks died in this group with a mortality rate of $57 \%$. Gross lesions were severe accumulation of fibrinous material on the heart, liver, and air sacs. In addition, surviving chicks also showed severe postmortem lesions affecting the heart, liver, and air sacs. In Group 5 (infected and treated with tigecycline), clinical signs were almost similar to that of the positive control group with 15 dead chicks (50\% mortality rate). The gross lesions in the dead and surviving chicks were

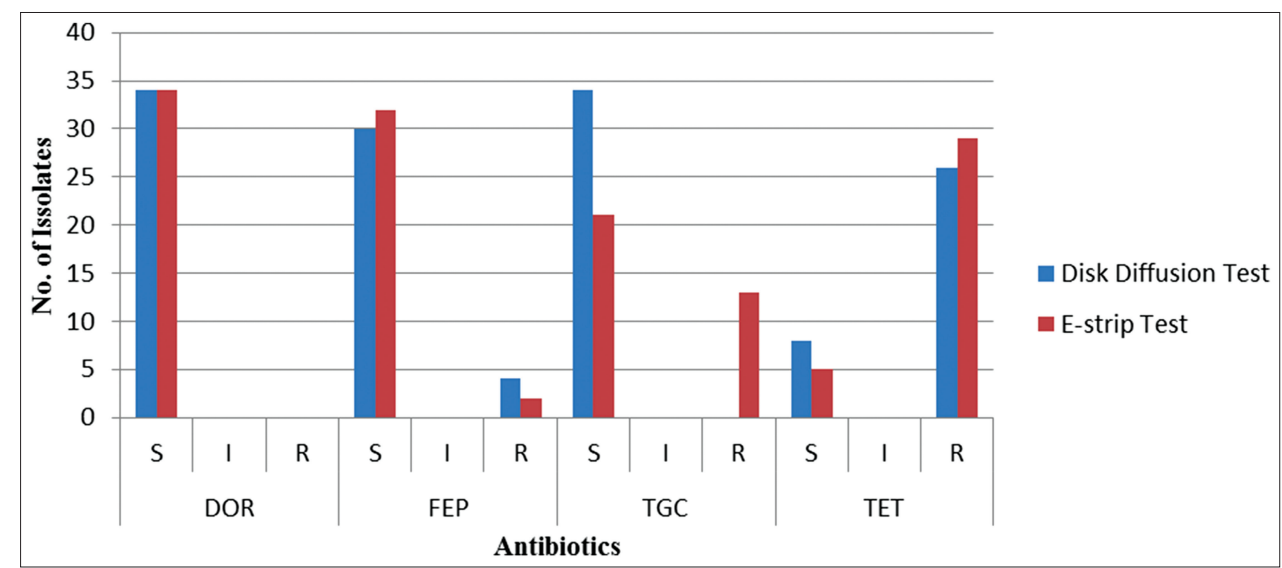

Figure-1: Antibiotic resistance profile of 34 isolates of extended-spectrum beta-lactamases producing Escherichia coli against cefepime, doripenem, tigecycline, and tetracycline. DOR=Doripenem, FEP=Cefepime, TGC=Tigecycline, TET=Tetracycline, $\mathrm{S}=$ Susceptible, I= Intermediate, $\mathrm{R}=$ Resistant. 


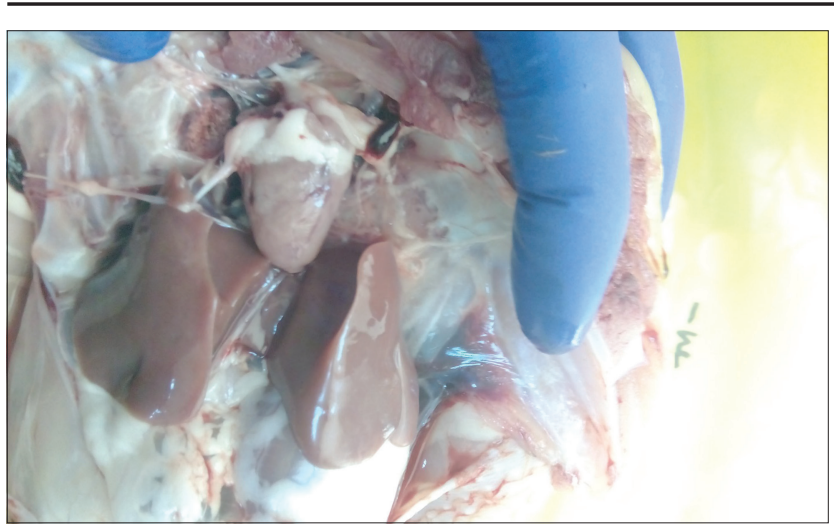

Figure-2: No gross lesions could be observed in nonchallenged control chicks.

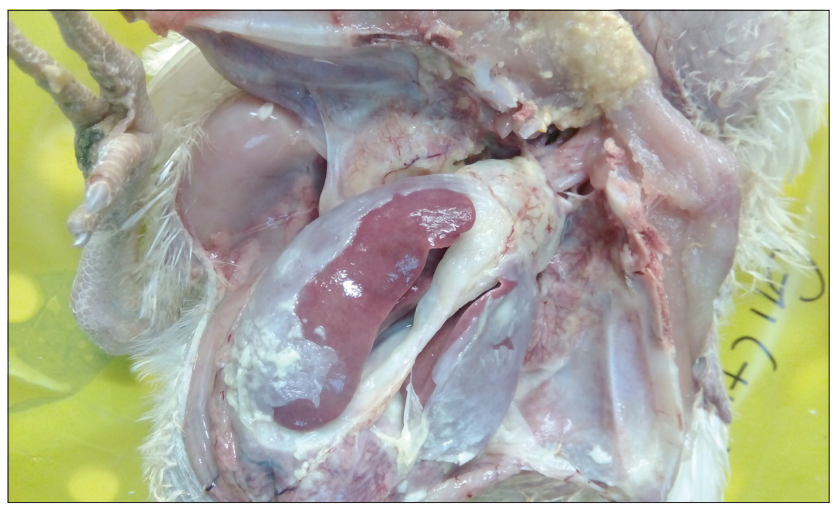

Figure-3: Infected chick with multidrug-resistantextended-spectrum beta-lactamases producing Escherichia coli with no treatment administered (positive control) showing severe fibrinous formation on the heart, liver, and air sacs.

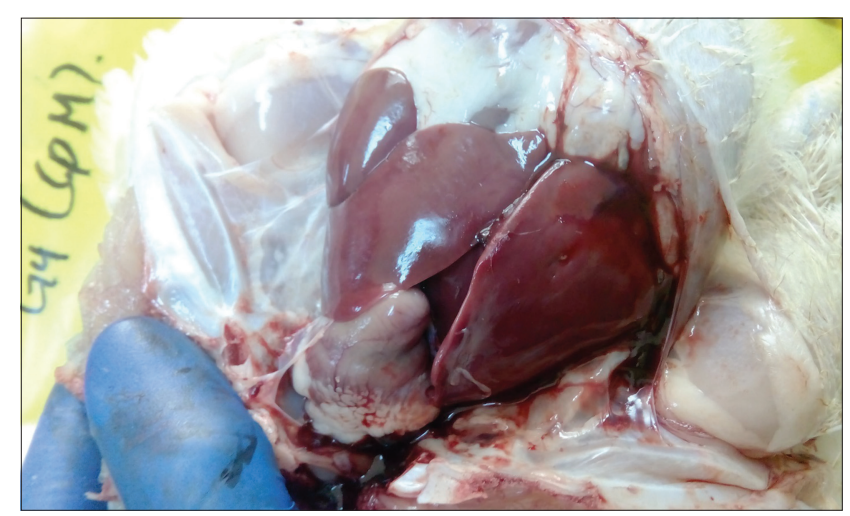

Figure-4: Infected chick with multidrug-resistantextended-spectrum beta-lactamases producing Escherichia coli treated with cefepime showing slightly congested liver with no to little accumulation of fibrinous materials on the heart, liver, and air sacs.

similar to those observed in the positive control group. In Group 6 (infected and treated with tetracycline), clinical signs and postmortem lesions were similar to those observed in the positive control group with a mortality rate of $90 \%$.

In general, the differences between the groups were significant $(\mathrm{p} \leq 0.05)$ when the comparison was made between treated groups and non-treated chicks (Table-1). However, statistical analysis of the lesion
Table-1: Mean ranks of gross lesions in different body organs in chicks infected with multidrug-resistant ESBLs producing $E$. coli.

\begin{tabular}{|c|c|c|c|c|c|c|}
\hline \multirow[t]{2}{*}{ Organs } & \multicolumn{6}{|c|}{ Groups } \\
\hline & 1 & 2 & 2 & 4 & 5 & 6 \\
\hline Liver & $144^{a}$ & $20^{\text {ăe }}$ & $105^{\text {ăè }}$ & $76^{\text {ăèc }}$ & 119 ăč & $132^{a}$ \\
\hline Heart & $145^{a}$ & $23^{\text {ăe }}$ & $103^{\text {ăē }}$ & 74 & $108^{\text {ăč }}$ & $140^{a}$ \\
\hline Air-sacs & $154^{a}$ & $27^{\text {ăe }}$ & $95^{\text {ăē }}$ & 74 ăềc & $104^{\text {ăč }}$ & $129^{a ̆}$ \\
\hline
\end{tabular}

aăIndicate significant difference at $p \leq 0.05$. eēIndicate significant difference at $p \leq 0.05$. č́Indicate significant difference at $\mathrm{p} \leq 0.05$. E. coli=Escherichia coli, MDR-ESBL=Multidrug-resistant extended-spectrum beta-lactamases

scores of visceral organs revealed that chicks in Group 4 (treated with cefepime) exhibited the lowest mean rank compared to all other groups.

\section{Discussion}

Treatments of many infectious diseases have become a great challenge due to the emergence of multidrug antibiotic resistance among pathogenic microorganisms [31]. Indeed, MDR microorganisms are now widespread in the environment and are posing serious threats to public health worldwide[32]. Commercial development of new classes of antibiotics has diminished over the past 15 years and few pharmaceutical companies remain active, which indicates an urgent need for new medications to overcome the rising problem of MDR microorganisms [32]. $\beta$-lactams remain the mainstay antibiotic therapeutic agents against many bacterial infections [24]. Unfortunately, injudicious use of these antimicrobial agents over the years rendered then ineffective $[9,33]$. Therefore, in this study, a new generation of antibiotics has been chosen to evaluate their efficacy against MDR-ESBLs producing E. coli in chickens.

Avian pathogenic E. coli (APEC) is associated with huge economic losses to the poultry industry due to high prevalence rates of multidrug resistance among this bacterium [34]. It has been demonstrated that most APEC isolated from broilers are resistant to sulfamethoxazole-trimethoprim, florfenicol, amoxicillin, doxycycline, spectinomycin, tetracycline, and erythromycin [34].

In this study, MDR-ESBLs producing E. coli were found highly sensitive to doripenem and cefepime showing the largest inhibitory zone among all tested antibiotics. In addition, results of the MIC breakpoints revealed that this microorganism is highly susceptible to both antibiotics. These results are in total agreement with previously published data $[25,35]$. In addition, six of the tested E. coli isolates (18\%) were found resistant to cefepime which is similar to the previous findings reported previously by Mansouri et al. [36]. Thirteen $E$. coli isolates were found resistant to tigecycline. This also was similar to the results obtained by Wang et al. [37]. Twenty-three E. coli isolates were 
found sensitive to tetracycline, which also similar to the results obtained by Kabiru et al. [28].

In this study, the challenge test aimed to determine the effectiveness of these antimicrobial agents on infections caused by MDR-ESBLs producing E. coli in broiler chicks. Induction of colisepticemia by inoculating E. coli (O78) directly to the air sac is an effective approach in producing clinical colibacillosis and colisepticemia in broiler chicks [38]. Colisepticemia usually occurs within 3-12 h after inoculation, through bacterial passage across the air capillary walls [38]. The pathogenicity of $E$. coli strain used in the challenge test in this study was previously determined [30]. The challenged bacteria were MDR-ESBLs producing E. coli serotype $\mathrm{O} 78$ which possess several virulence-associated genes obtained from broiler chickens suffering from chronic respiratory disease [39]. In the challenged chicks, the clinical signs and gross lesions in nontreated chicks were consistent with severe colibacillosis. The mortality rate was up to $55 \%$ in this group. In chicks that were treated using different antibiotics, the clinical signs, gross lesions, and mortality rates were variable compared to the control groups. For example, in doripenem- and tigecycline-treated chicks, the mortality rates were 57 and $50 \%$, respectively. This indicates that doripenem and tigecycline were not effective for the treatment of infection caused by this strain of $E$. coli. Although, both of these antibiotics showed 100\% susceptibility against $E$. coli in the in vitro study. The difference between in vitro susceptibility of antibiotics and in vivo efficacy is commonly encountered in clinical practice. This can only be explained by performing specialized biokinetic studies. However, similar results have been obtained previously by Samonis et al. [40].

In challenged chicks treated by tetracycline, the mortality rate was $90 \%$. In the in vitro susceptibility test, $77 \%$ of $E$. coli isolates were resistant to tetracycline in vitro. This means that tetracycline is not an effective drug for the treatment of colibacillosis in broiler chicks. The high resistant profile of E. coli against tetracycline is similar to the results of Al-Bahry et al. [41]. In challenged chicks treated with cefepime, the mortality rate was only $3 \%$. The in vitro susceptibility test demonstrated that $88 \%$ of the tested $E$. coli isolates were susceptible to cefepime. In fact, cefepime-treated chicks showed obvious improvement in clinical signs and reduction in the scores of gross lesions and a significant $(\mathrm{p} \leq 0.05)$ reduction in mortality rate in comparison to those in the untreated control group. These results are similar to previously reported findings in broiler chickens [42].

\section{Conclusion}

The results obtained from this study indicated that cefepime is an effective antimicrobial agent against infections caused by MDR-ESBLs producing E. coli in broiler chicks. Therefore, this antibiotic could be used in the treatment of naturally occurring colibacillosis in chickens.

\section{Authors' Contributions}

YHT designed the project protocol, supervised the whole work, and wrote the manuscript. EAA and ZBI involved in the manuscript writing and supervised the pathogenicity and challenge assay. RAT did the laboratory works. All authors read and approved the final manuscript.

\section{Acknowledgments}

This study was funded by the Deanship of Research at Jordan University of Science and Technology project \# 20190048

\section{Competing Interests}

The authors declare that they have no competing interests.

\section{Publisher's Note}

Veterinary World remains neutral with regard to jurisdictional claims in published institutional affiliation.

\section{References}

1. Prestinaci, F., Pezzotti, P. and Pantosti, A. (2015) Antimicrobial resistance: A global multifaceted phenomenon. Pathog. Glob. Health., 109(7): 309-318.

2. Aslam, B., Wang, W., Arshad, M.I., Khurshid, M., Muzammil, S., Rasool, M.H., Nisar, M.A., Alvi, R.F., Aslam, M.A., Qamar, M.U., Salamat, M.K.F. and Baloch, Z. (2018) Antibiotic resistance: A rundown of a global crisis. Infect. Drug Resist., 10(11): 1645-1658.

3. Tomley, F.M. and Shirley, M.W. (2009) Livestock infectious diseases and zoonoses. Philos. Trans. R. Soc. Lond. B. Biol. Sci., 364(1530): 2637-2642.

4. Daikos, G.L., Tsaousi, S., Tzouvelekis, L.S., Anyfantis, I., Psichogiou, M., Argyropoulou, A., Stefanou, I., Sypsa, V., Miriagou, V., Nepka, M., Georgiadou, S., Markogiannakis, A., Goukos, D. and Skoutelis, A. (2014) Carbapenemase-producing Klebsiella pneumoniae bloodstream infections: Lowering mortality by antibiotic combination schemes and the role of carbapenems. Antimicrob. Agents Chemother., 58(4): 2322-2328.

5. Russo, T.A. and Johnson, J.R. (2003) Medical and economic impact of extraintestinal infections due to Escherichia coli: Focus on an increasingly important endemic problem. Microbes Infect., 5(5): 449-456.

6. Rasheed, M.U., Thajuddin, N., Ahamed, P., Teklemariam, Z. and Jamil, K. (2014) Antimicrobial drug resistance in strains of Escherichia coli isolated from food sources. Rev. Inst. Med. Trop. Sao Paulo, 56(4): 341-346.

7. Allocati, N., Masulli, M., Alexeyev, M.F. and Di Ilio, C. (2013) Escherichia coli in Europe: An overview. Int. J. Environ. Res. Public Health, 10(12): 6235-6254.

8. Li, B. and Webster, T.J. (2018) Bacteria antibiotic resistance: New challenges and opportunities for implant-associated orthopedic infections. J. Marriage Fam., 57(2): 283-294

9. Singh, N., Madhu, M., Vanamail, P., Malik, N. and Kumar, S. (2017) Study of adverse drug reactions in patients with diabetes attending a tertiary care hospital in New Delhi, India. Indian J. Med. Res., 145(8): 247-249.

10. Hur, J., Jawale, C. and Lee, J.H. (2012) Antimicrobial resistance of Salmonella isolated from food animals: A review. Food Res. Int., 45(2): 819-830.

11. Harada, K., Shimizu, T., Mukai, Y., Kuwajima, K., Sato, T. and Kataoka, Y. (2016) Phenotypic and molecular characterization of antimicrobial resistance in Klebsiella spp. 
isolates from companion animals in Japan: Clonal dissemination of multidrug-resistant extended-spectrum $\beta$-lactamase-producing Klebsiella pneumoniae. Front. Microbiol., 7(7): 1-12.

12. Davis, G.S., Waits, K., Nordstrom, L., Weaver, B., Aziz, M., Gauld, L. and Price, L.B. (2015) Intermingled Klebsiella pneumoniae populations between retail meats and human urinary tract Infections. Clin. Infec. Dis., 61(6): 892-899.

13. Guo, Y., Zhou, H., Qin, L., Pang, Z., Qin, T., Ren, H. and Zhou, J. (2016) Frequency, antimicrobial resistance and genetic diversity of Klebsiella pneumoniae in food samples. PLoS One, 11(4): 1-13.

14. Podder, M.P., Rogers, L., Daley, P.K., Keefe, G.P., Whitney, H.G. and Tahlan, K. (2014) Klebsiella species associated with bovine mastitis in Newfoundland. PLoS One, 9(9): 1-5.

15. Aloush, V., Navon-Venezia, S., Seigman-Igra, Y., Cabili, S and Carmeli, Y. (2006) Multidrug-resistant Pseudomonas aeruginosa: Risk factors and clinical impact. Antimicrob. Agents Chemother., 50(1): 43-48.

16. Hirsch, E.B. and Tam, V.H. (2010) Impact of multidrug-resistant Pseudomonas aeruginosa infection on patient outcomes. Expert Rev. Pharmacoecon. Outcomes Res., 66(3): 441-451.

17. Katiyar, C., Gupta, A., Kanjilal, S. and Katiyar, S. (2012) Drug discovery from plant sources: An integrated approach. Ayu, 33(1): 10-19.

18. Stein, G.E. and Babinchak, T. (2013) Tigecycline: An update. Diagn. Microbiol. Infect. Dis., 75(4): 331-336.

19. Garrison, M.W., Neumiller, J. and Setter, S.M. (2005) Tigecycline: An investigational glycylcycline antimicrobial with activity against resistant gram-positive organisms. PLoS One, 27(1): 25-73.

20. Patel, H.B., Lusk, K.A. and Cota, J.M. (2017) The role of cefepime in the treatment of extended-spectrum beta-lactamase infections. J. Pharm. Pract., 55(2): 25-29.

21. Siedner, M.J., Galar, A., Guzmán-Suarez, B.B., Kubiak, D.W., Baghdady, N., Ferraro, M.J. and Marty, F.M. (2014) Cefepime vs other antibacterial agents for the treatment of Enterobacter species bacteremia. Clin. Infec. Dis., 58(11): 1554-1563.

22. Spellberg, B. and Gilbert, D.N. (2014) The future of antibiotics and resistance: A tribute to a career of leadership by John Bartlett. Clin. Infect. Dis., 59(2): S71-S75.

23. Clinical and Laboratory Standards Institute. (2011) Performance Standards for Antimicrobial Susceptibility Testing; $21^{\text {st }}$ Informational Supplement. $3^{\text {rd }}$ ed., Vol. 31. Clinical and Laboratory Standards Institute, Wayne, PA.

24. Wani, K.A., Thakur, M.A., Fayaz, S.A., Fomdia, B., Gulnaz, B. and Maroof, P. (2009) Extended-spectrum B-lactamase mediated resistance in Escherichia coli in a tertiary care hospital. Int. J. Health Sci., 3(2): 155-163.

25. Walsh, F. (2007) Doripenem: A new carbapenem antibiotic a review of comparative antimicrobial and bactericidal activities. Ther. Clin. Risk Manag., 3(5): 789-794.

26. Troy, S., Muralidharan, G., Micalizzi, M., Speth, J. and Raible, D. (2005) Pharmacokinetics of tigecycline after single and multiple doses in healthy subjects. Am. Soc. Microbiol., 78(1): 67-79.

27. Calandra, D.M., Mauro, D.D., Cutugno, F. and Martino, S.D. (2016) Navigating wall-sized displays with the gaze: A proposal for cultural heritage. CEUR Workshop Proc., 1621(9):
36-43.

28. Kabiru, L.M., Bello, M., Kabir, J., Grande, L. and Morabito, S. (2015) Detection of pathogenic Escherichia coli in samples collected at an abattoir in Zaria, Nigeria and at different points in the surrounding environment. Int. $J$. Environ. Res. Public Health, 12(1): 679-691.

29. Shah, S.A.A., Akhter, N., Auckloo, B.N., Khan, I., Lu, Y., Wang, K., Wu, B. and Guo, Y. (2017) Structural diversity, biological properties and applications of natural products from cyanobacteria. A review. Mar. Drugs, 15(11): 354.

30. Van der Waaij, E.H. (2017) Escherichia coli pathogenicity and lesions in broiler chickens in Spain. Vet. Rec., 128(7): 244-258.

31. Wang, Z., Qin, R.R., Huang, L. and Sun, L.Y. (2018) Risk factors for carbapenem-resistant Klebsiella pneumoniae infection and mortality of Klebsiella pneumoniae Infection. Chin. Med. J., 131(1): 56-59.

32. Müller, P., Alber, D.G., Turnbull, L., Schlothauer, R.C., Carter, D.A., Whitchurch, C.B. and Harry, E.J. (2013) Synergism between medihoney and rifampicin against methicillin-resistant Staphylococcus aureus (MRSA). PLoS One, 8(2): 1-9.

33. Erdem, H., Kiliç, S. and Pahsa, A. (2013) Gram-negative bacterial resistance to cephalosporins in community-acquired infections in Turkey. J. Chemother., 17(2): 2-15.

34. Ito, A., Hirai, K., Innoue, M., Koga, H., Suzue, S., Irikura, T. and Mitsuhash, S. (1980) In vitro antibacterial activity of AM-715, a new nalidixic acid analog. Antimicrob. Agents Chemother., 17(2): 103-107.

35. Martinez, J.L. (2014) General principles of antibiotic resistance in bacteria. Drug Discov. Today Technol., 11(2): 33-39.

36. Mansouri, M., Ramazanzadeh, R. and Norabadi, P. (2011) Cefepime resistance and associated risk factors among Escherichia coli strains isolated from clinical specimens. Chemotherapy, 57(2): 134-137.

37. Wang, Q., Zhang, P., Zhao, D., Jiang, Y., Zhao, F., Wang, Y., Li, X., Du, X. and Yu, Y. (2018) Emergence of tigecycline resistance in Escherichia coli co-producing MCR-1 and NDM-5 during tigecycline salvage treatment. Infect. Drug Resist., 13(11): 2241-2248.

38. Lowbury, E.J.L., Thom, B.T., Lily, B.T.J., Babb, R. and Wittal, K. (2010) Source of infection with Pseudomonas aeruginosa in patients with tracheotomy. J. Clin. Microbiol., 3(3): 39-56.

39. Ibrahim, R.A., Cryer, T.L., Lafi, S.Q., Abu Basha, E., Good, L. and Tarazi, Y.H. (2019) Identification of Escherichia coli from broiler chickens in Jordan, their antimicrobial resistance, gene characterization and the associated risk factors. BMC Vet. Res., 15(5): 159-161.

40. Samonis, G., Maraki, S., Karageorgopoulos, D.E., Vouloumanou, E.K. and Falagas, M.E. (2012) Synergy of fosfomycin with Klebsiella pneumoniae, Escherichia coli, and Pseudomonas aeruginosa clinical isolates. Eur. J. Clin. Microbiol. Infect. Dis., 31(4): 695-701.

41. Al-Bahry, S.N., Al-Mashani, B.M., Al-Ansari, A.S., Elshafie, A.E. and Mahmoud, I.Y. (2013) Escherichia coli tetracycline efflux determinants in relation to tetracycline residues in chicken. Asian Pac. J. Trop. Med., 6(3): 718-722.

42. Morita,Y., Tomida, J. and Kawamura, Y. (2013) Responses of Pseudomonas aeruginosa to antimicrobials. Front. Microbiol., 4(3): 422-428. 